\title{
Advanced Soft Magnetic Materials for Magnetic Recording Heads and Integrated Inductors
}

N. X. Sun ${ }^{1}$, A. M. Crawford, and S. X. Wang

Dept. of Materials Science and Engineering, Stanford University, Stanford, CA 94305-4045

${ }^{1}$ Presently at IBM Storage Technology Division, San Jose, CA 95193

\section{ABSTRACT}

High performance magnetic heads, inductors and transformers, indispensable to information technology encompassing from information storage, portable power delivery, to wireless communication, require soft magnetic films with low coercivities, high permeability, and large ferromagnetic resonance frequencies.

The Fe-Co-N-based films have a ferromagnetic resonance frequency of $>1 \mathrm{GHz}$ at zerobias field, showing great promise for applications in write heads and integrated inductors in a frequency range of $>1 \mathrm{GHz}$. Magnetization dynamics measurements at sub-nanosecond scale have been performed on Fe-Co-N high saturation soft magnetic films with Permalloy nanolayer seeds having a saturation magnetization of $24 \mathrm{kG}$. The high frequency behavior appears to be affected by magnetic anisotropy dispersion.

One of the biggest challenges facing integration of magnetic material onto silicon is the compatibility of magnetics with standard silicon processing techniques. Integrated inductors were realized using ground planes of $\mathrm{Co}-\mathrm{Ta}-\mathrm{Zr}(\rho=100 \mu \Omega-\mathrm{cm})$. The magnetic properties of Co$\mathrm{Ta}-\mathrm{Zr}$ showed no change even after undergoing high temperature processing. Inductors with $1 \mu \mathrm{m}$ Co-Ta-Zr produced inductance values up to $60 \%$ higher than the air core inductors at frequencies up to $1.4 \mathrm{GHz}$.

\section{INTRODUCTION}

Soft magnetic films with low coercivity and high permeability are critical building blocks in numerous electromagnetic devices such as magnetic recording heads, integrated inductors (microinductors), integrated transformers, magnetic sensors, and micromachined motors. However, a lack of soft magnetic materials with a high saturation magnetization $(>20 \mathrm{kG})$ and a large permeability roll-off frequency $(>1 \mathrm{GHz})$ often becomes the bottleneck in these applications, particularly in magnetic hard disk drives.

The arrival of the information age is made possible by faster and faster microprocessors, networks, and magnetic hard disk drives. It is anticipated that the data rate of hard disk drives will be $>1 \mathrm{~Gb} / \mathrm{s}(1 \mathrm{~Gb}=1$ billion bits) soon. A data rate of $2 \mathrm{~Gb} / \mathrm{s}$ corresponds to recording frequencies of $\sim 1 \mathrm{GHz}$, which exceeds the ferromagnetic resonance (FMR) frequency of most magnetic materials. In addition to the rapid increase in data rate, magnetic information storage technology is approaching the perceived superparamagnetic limit at which the stored bits become thermally unstable [1]. To ensure the stability of recorded information for 10 years will require the stability ratio of magnetic medium, $K_{u} V / k_{B} T$, to be 60-70 [2], where $K_{u}$ is the anisotropy energy density, $V$ is the magnetic switching volume, $k_{B}$ is the Boltzmann constant, and $T$ is the absolute temperature. The estimated maximum areal bit density of conventional longitudinal magnetic recording [3] ranges from 40 to $150 \mathrm{~Gb} / \mathrm{in}^{2}$, depending on the assumptions of magnetic 
medium thickness and relevant materials parameters. In fact, the superparamagnetic limit is a writing problem more than a medium problem or a reading problem. Many high $K_{u}$ media are available for accomodating areal densities $>100 \mathrm{~Gb} / \mathrm{in}^{2}$, but they are not practical because magnetic write heads cannot magnetically saturate them unless exotic schemes like thermally assisted writing are employed [2]. Rapid development in giant magnetoresistive (GMR) materials and spin-dependent devices [4,5] have enabled the read heads to detect tiny bits even though we do not know how to write them. The writing problem arises from the very limited choices of soft magnetic materials that can be used in magnetic write heads.

As manifested by the Slater-Pauling curve [6], the maximum saturation magnetization available in the nature is $\sim 24.5 \mathrm{kG}$, found in $\mathrm{Fe}_{1-\mathrm{x}} \mathrm{Co}_{\mathrm{x}}$ alloys $(0.3 \leq \mathrm{x} \leq 0.4)$. Unfortunately, the FeCo alloys are highly magnetostrictive, and their saturation magnetostriction constant (ranging from $40 \times 10^{-6}$ to $65 \times 10^{-6}$ ) is about two orders of magnitude greater than that of Permalloy $\left(\mathrm{Ni}_{.81} \mathrm{Fe}_{.19}\right)$. As a result, the coercivity of the FeCo alloys tends to be well above 5 Oe [7], making them unusable for magnetic write heads. Past experiments have identified soft magnetic materials such as $\mathrm{Co}_{.57} \mathrm{Ni}_{.13} \mathrm{Fe}_{30}$ [8], and $\mathrm{FeXN}$ [9], where $\mathrm{X}=\mathrm{Al}$, Ta, Rh, Mo, Si, Zr, etc., for high saturation write head applications, but their saturation magnetization is $\sim 20 \mathrm{kG}$. Therefore, it is a significant development to make soft magnetic films based on Fe-Co-N with a saturation magnetization of $\sim 24 \mathrm{kG}[10]$.

Unlike information storage, the microelectronics industry is not limited by the superparamagnetic effects, but it can also reap great benefits by incorporating high saturation soft magnetic materials into integrated inductors. An inductor (or transformer) with an appropriate soft magnetic film as its core material can be made smaller, cheaper, less power hungry, more efficient, and more portable than its counterpart with an air coil. A higher saturation magnetic core will allow a larger drive current in the inductor (or transformer), even though it is not always necessary like in the magnetic write heads.

Generally speaking, the requirements of the soft magnetic films for inductor and magnetic head applications are very similar, as listed in Table I. In this paper, we will describe two types of materials, Fe-Co-N and Co-Ta-Zr, with their saturation magnetization being $24 \mathrm{kG}$ and $15 \mathrm{kG}$, respectively. Both are suitable for magnetic write heads and integrated inductors, and we have made high frequency inductors using the latter material because of its high resistivity $(\sim 100 \mu \Omega \mathrm{cm})$ and thermal stability (up to $400{ }^{\circ} \mathrm{C}$ ).

Table I. Desired properties of magnetic head pole and inductor core materials.

\begin{tabular}{|l|l|l|}
\hline Desired Properties & Magnetic Write Head & Inductor \\
\hline Coercivity & $\sim 1$ Oe or smaller & $\sim 1$ Oe or smaller \\
\hline Permeability & $\sim 500$ or higher & $\sim 100$ or higher \\
\hline Operation Frequency & $\sim 250 \mathrm{MHz}$ or higher & $\sim 1 \mathrm{MHz}-10 \mathrm{GHz}$ \\
\hline Saturation Magnetization & $>20 \mathrm{kG}$ & depending on applications \\
\hline Magnetic Anisotropy & Uniaxial & Uniaxial \\
\hline Resistivity & $>20 \mu \Omega \mathrm{cm}$ & $>100 \mu \Omega \mathrm{cm}$ \\
\hline Processing Temperature & $\sim 250{ }^{\circ} \mathrm{C}$ (max) & $\sim 400{ }^{\circ} \mathrm{C}$ (max) \\
\hline Corrosion Resistance & Exposed to ambient & Can be passivated \\
\hline Magnetostriction Constant & As small as possible & As small as possible \\
\hline
\end{tabular}




\section{Fe-Co-N HIGH SATURATION MATERIALS FOR MAGNETIC HEADS AND INDUCTORS}

We have developed Fe-Co-N based materials with Permalloy nanolayer seeds that are very promising for applications as write head pole and inductor core materials. Their properties are summarized in Table II, where $B_{\mathrm{s}}$ is the saturation flux density, $H_{\mathrm{c}}$ the hard axis coercivity, $H_{\mathrm{k}}$ the in-plane anisotropy field, $\rho$ the resistivity, $\chi_{0}$ the initial susceptibility along the hard axis, and $f_{3 \mathrm{~dB}}$ the $3 \mathrm{~dB}$ roll-off frequency of the real part of the hard axis permeabilty. The latter is basically determined by the ferromagnetic resonance (FMR) frequency, which should normally be above the operation frequency of magnetic heads (or inductors). The details regarding the fabrication and soft magnetism of these materials have been published elsewhere [10,11].

Table II. Properties of Fe-Co-N films with Permalloy nanolayer seeds.

\begin{tabular}{|l|l|l|l|l|l|}
\hline$B_{\mathrm{s}}(\mathrm{T})$ & $H_{\mathrm{c}}(\mathrm{A} / \mathrm{m})$ & $H_{\mathrm{k}}(\mathrm{kA} / \mathrm{m})$ & $\chi_{0}$ & $\rho(\mu \Omega \cdot \mathrm{cm})$ & $f_{3 \mathrm{~dB}}(\mathrm{GHz})$ \\
\hline 2.4 & $<80$ & 1.6 & 1200 & 50 & 1.5 \\
\hline
\end{tabular}

With the continued increase of data rates in magnetic disk drives, the high-frequency performance of soft magnetic thin films becomes increasingly important [3-5]. Coherent rotation of the magnetization is desired for applications of magnetic films because it corresponds to the fastest switching of magnetization. In practice, due to the local variation of the anisotropy axes over the whole soft film area, coherent rotation will only occur when the angle between the applied field and the easy axis is less than $\left(90^{\circ}-\alpha\right)$ at low frequencies, where $\alpha$ is the dispersion angle [12]. The dispersion angle $\alpha$ is easily measured by a standard B-H looper, and is typically less than $1^{\circ}$ for soft magnetic films with a good uniaxial anisotropy. How the dispersion angle affects the high frequency behavior is still not well understood and is under investigation.

Fe-Co-N films with a thickness of $100 \mathrm{~nm}$ and with Permalloy nano-underlayer $(<5 \mathrm{~nm})$ have been synthesized with a high saturation magnetization of $24 \mathrm{kG}$, an anisotropy field of about 20 Oe, and a coercivity of less than 1 Oe [10,11]. The Fe-Co-N films show a high initial permeability of 1000 with a bandwidth of $1.5 \mathrm{GHz}$. The Fe-Co-N films with Permalloy nanounderlayer (in short Py $\backslash \mathrm{Fe}-\mathrm{Co}-\mathrm{N}$ ) show a drastic improvement in the magnetic softness compared to the single layer Fe-Co-N film. For all the Py $\backslash$ Fe-Co-N films, the Fe-Co-N layer thickness was fixed at $100 \mathrm{~nm}$ and the Permalloy underlayer thickness was varied in the range of $0 \mathrm{~nm}$ to $5 \mathrm{~nm}$. In this paper, the high frequency behavior of the Fe-Co-N single layer films and the Py $\backslash \mathrm{Fe}-\mathrm{Co}-\mathrm{N}$ films, which have drastically different dispersion angles but otherwise similar properties, will be presented and compared.

The Fe-Co-N films were deposited onto $0.5 \mathrm{~mm}$ thick Si wafers with a resistivity of 10$20 \Omega \cdot \mathrm{cm}$ through reactive RF diode sputtering in an argon and nitrogen atmosphere. The target composition was $\mathrm{Fe}_{70} \mathrm{Co}_{30}(\mathrm{at} \%)$ with a purity of $99.95 \%$. The Permalloy underlayer was deposited in a pure argon atmosphere by RF sputtering with a target composition of $\mathrm{Ni}_{81} \mathrm{Fe}_{19}$. The base pressure of the sputtering chamber was $\sim 2 \times 10^{-7}$ Torr. Magnetic properties of the $\mathrm{Py} \backslash \mathrm{Fe}-\mathrm{Co}-\mathrm{N}$ films were characterized by a standard B-H looper. The dispersion angles of magnetic films were measured with the B-H looper by using the following method [12]. By applying a large $\mathrm{AC}$ field $H_{\sim}\left(\right.$ larger than $H_{k}$ ) perpendicular to the easy axis, without a small DC 
field $H_{\mathrm{DC}}$ parallel to the easy axis, there will be no signal in the pick-up loop which is perpendicular to the easy axis since the magnetization falls to the easy axis symmetrically. When a DC magnetic field $H_{\mathrm{DC}}=H_{\mathrm{k}} \cdot \sin \alpha$ is applied along the easy axis, where $\alpha$ is the dispersion angle, a maximum signal in the detection coil will occur since all the local magnetization will fall to the easy axis. If $H_{\mathrm{DC}}$ must be reduced to $H_{D C}(50 \%)$ to obtain $50 \%$ of the maximum signal, we can get the $\alpha_{50}$ as

$$
\alpha_{50}=\frac{H_{D C}(50 \%)}{H_{k}} .
$$

The dispersion angles $\alpha_{50}$ of the Py|Fe-Co-N films can be tuned by changing the Permalloy underlayer thickness, as indicated in Fig. 1, being $2^{\circ}$ for the Fe-Co-N single layer film, and $0.8^{\circ}$ for the Py|Fe-Co-N film with a Permalloy underlayer thickness of $2.5 \mathrm{~nm}$. The saturation magnetization $B_{s}$ of the PylFe-Co-N film is very close to that of the single layer Fe$\mathrm{Co}-\mathrm{N}$ film. The Fe-Co-N single layer and the PylFe-Co-N film serve as excellent model specimens for studying the dispersion angle effects on the high frequency behavior of magnetic films.

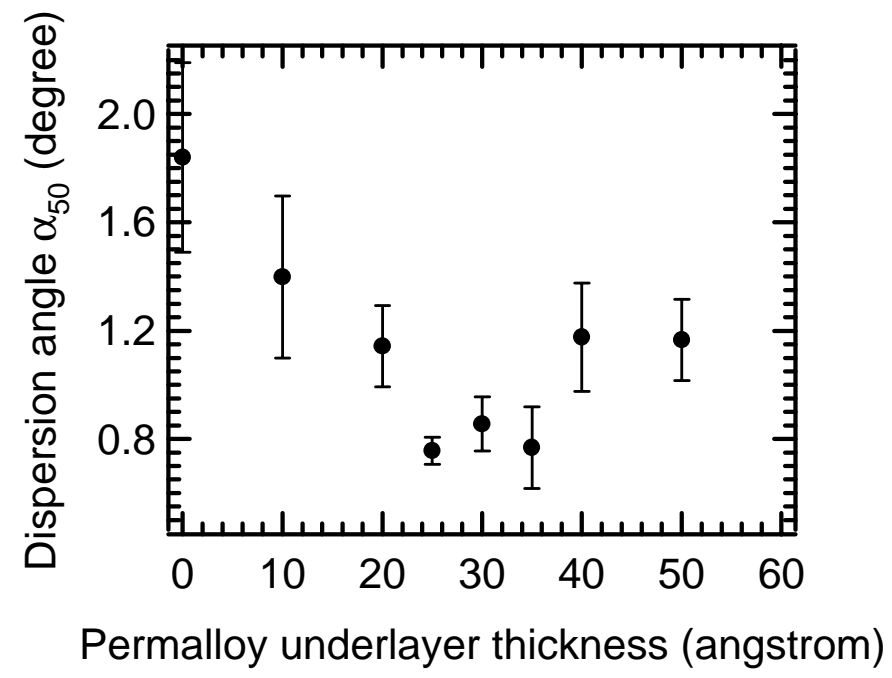

Figure 1. Dispersion angle $\alpha_{50}$ of the Py|Fe-Co-N films with different Permalloy underlayer thicknesses, with the error bar showing the range of data scattering.

A pulsed inductive microwave magnetometer (PIMM) was used to study the high frequency behavior of the Py|Fe-Co-N films [13,14]. The PIMM comprises a pulse generator and an oscilloscope connected by a waveguide, on which the magnetic film is placed. The whole system has a bandwidth of $6 \mathrm{GHz}$. Two sets of biasing Helmholtz coils provide the DC magnetic fields parallel and perpendicular to the waveguide direction. The electrical pulse produces an excitation field perpendicular to the waveguide according to Ampere's law. The magnetic film on the waveguide responds to the excitation, and the inductive voltage generated by the timevarying magnetization along the excitation direction is recorded by the oscilloscope in the time domain. The detected magnetization signal can be fitted by the following equation to get the damping constant $\alpha$. 


$$
\phi(t)=\phi_{0}+\beta_{0} \sin \left(\omega_{p} t+\varphi\right) e^{-\frac{\eta_{0} M_{s} \alpha t}{2}}
$$

with $\phi(t)$ being the in-plane angle of the magnetization, $\phi_{0}$ the equilibrium angle which is equal to 0 for impulse responses, $\omega_{\mathrm{p}}$ the ferromagnetic resonance (FMR) frequency, $\gamma$ the gyromagnetic constant, and $\mu_{0}$ the vacuum permeability. The inductive time-domain data were transformed to the frequency domain to obtain the permeability spectrum. The ferromagnetic resonance (FMR) frequency was taken as the peak frequency of the imaginary permeability spectrum. In this work, the amplitude of the step magnetic field is chosen to be about $2.5 \mathrm{Oe}$, which is much smaller than the anisotropy field of 20 Oe. The magnetization oscillates with an angular amplitude of less than $130 \mathrm{mrad}$ under such an excitation field, satisfying the smallsignal condition.

The PIMM signals from the Fe-Co-N single layer film and the Py|Fe-Co-N show the typical exponentially decreasing sinusoidal curves. The Landau-Lifshitz damping constants are obtained from the time domain curves and shown in Fig. 2 as a function of the longitudinal bias field. The Py $\backslash \mathrm{Fe}-\mathrm{Co}-\mathrm{N}$ film with a low dispersion angle $\alpha_{50}$ of $0.8^{\circ}$ shows a much lower damping constant than the Fe-Co-N single layer film with a dispersion angle $\alpha_{50}$ of $2^{\circ}$ at all bias fields. At zero longitudinal bias field, the Py $\backslash \mathrm{Fe}-\mathrm{Co}-\mathrm{N}$ film shows a damping parameter of 0.019 ; while $\mathrm{Fe}-$ Co-N film shows a much larger damping parameter of 0.027 . The damping constants drop with the bias fields at low bias fields, but increase with the longitudinal bias field when the bias field is larger than $40 \mathrm{Oe}$. This is believed to be related to the microwave absorption of the $\mathrm{Si}$ substrate at high frequencies.

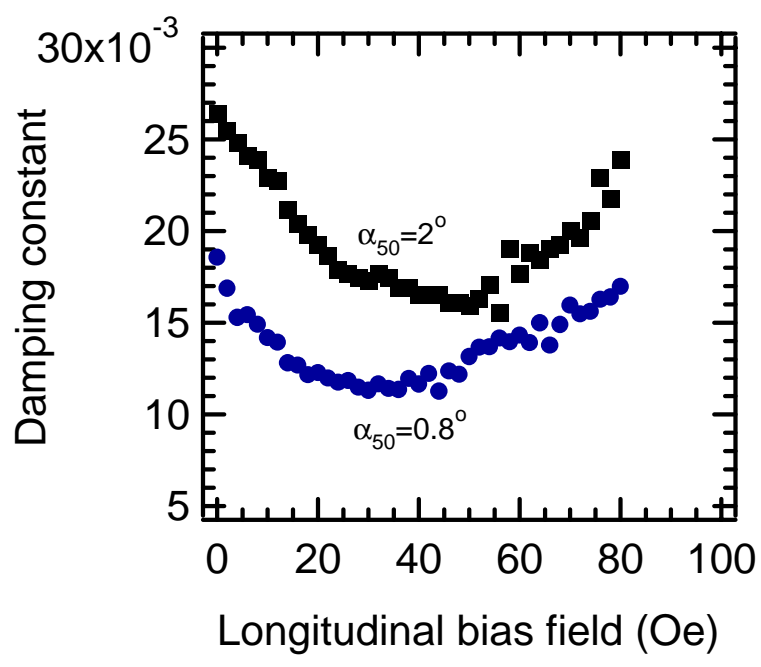

Figure 2. Landau-Lifshitz damping parameters for the two Fe-Co-N films with different dispersion angles as a function of the longitudinal bias function with $\mathrm{AC}$ excitation field amplitude of $8 \mathrm{~A} / \mathrm{m}(0.1 \mathrm{Oe})$.

The FMR frequencies are shown in Fig. 3 as a function of the longitudinal bias field for both the Fe-Co-N film and the Py $\backslash \mathrm{Fe}-\mathrm{Co}-\mathrm{N}$ film. The FMR frequencies increase continuously with the longitudinal bias field due to the increase of the net magnetic field in the film when longitudinally biased. Clearly the Py|Fe-Co-N film with a dispersion angle of $0.8^{\circ}$ shows higher 
FMR frequencies at all bias fields than the single layer Fe-Co-N film that has a high dispersion angle of $2^{\circ}$. The FMR frequency at zero bias field is $2.0 \mathrm{GHz}$ for the PylFe-Co-N film, which makes the Py $\backslash \mathrm{Fe}-\mathrm{Co}-\mathrm{N}$ film a very promising candidate for high frequency applications such as magnetic write heads. The single layer Fe-Co-N film shows an FMR frequency of $1.7 \mathrm{GHz}$ at zero longitudinal bias field.

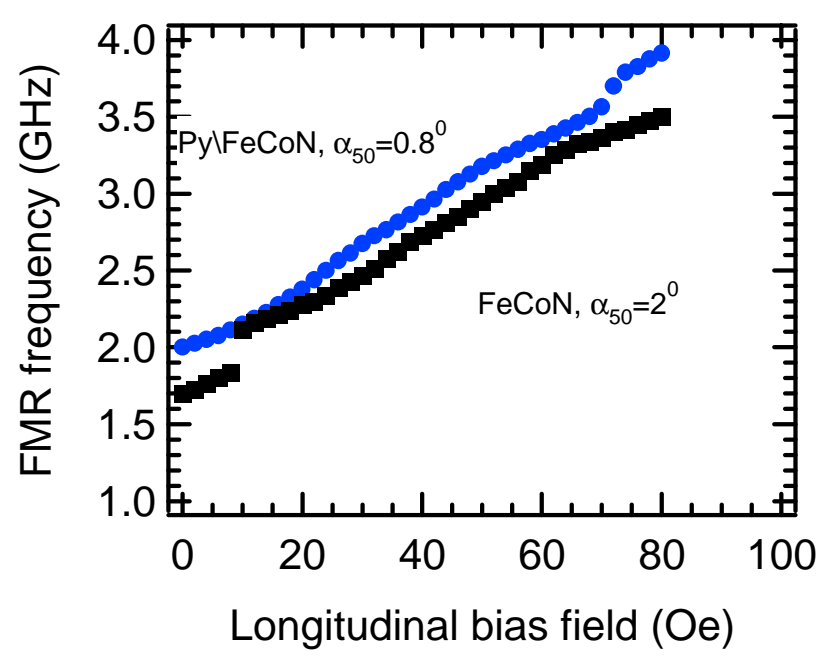

Figure 3. Comparison of the peak FMR frequencies from a single layer Fe-Co-N film that has a dispersion angle $\alpha_{50}$ of $2^{\circ}$, and a Py|Fe-Co-N film that has a dispersion angle $\alpha_{50}$ of $0.8^{\circ}$, as a function of the longitudinal bias field. (The discontinuity at a bias of $10 \mathrm{Oe}$ is not well understood at this writing.)

In the case of a small excitation signal, coherent magnetization rotation, and negligibly small damping constant, the FMR frequencies $f_{0}$ of a magnetic thin film at a longitudinal bias field $H_{\text {appl }}$ can be described by the Kittel equation:

$$
f_{F M R}=(\gamma / 2 \pi) \mu_{0} \sqrt{M_{s}\left(H_{k}+H_{\text {appl }}\right)},
$$

where $\gamma=g \mu_{B} / \hbar$ is the gyromagnetic ratio and equal to $2 \pi \times 28 \times 10^{9} \mathrm{rad} /(\mathrm{s} \cdot \mathrm{T}), g$ is the Landé factor, $\mu_{B}$ is the Bohr magneton, $\hbar$ is Planck's constant, and $M_{s}$ is the saturation magnetization [15]. For the Fe-Co-N film and the PylFe-Co-N film with very close $M_{s}$, the anisotropy field is the only parameter that may bring difference to the FMR frequency according to the Kittel equation. The anisotropy fields of the two films, which are measured by extrapolating the minor loop measured on a B-H looper, are shown in Fig. 4 as a function of the drive field amplitude. The Fe-Co-N film does not show closed linear loops in all drive fields, suggesting a not welldefined effective anisotropy field for the Fe-Co-N film. The measured anisotropy fields for the two films deviate significantly at low drive fields, but converge gradually to an anisotropy field of $18 \mathrm{Oe}$ at large drive fields. This is close to the $H_{\mathrm{k}}$ value extrapolated from the FMR frequencies for the Py|Fe-Co-N film, 20 Oe at low bias fields and 16 Oe at large bias fields [14]. By assuming an $4 \pi M_{s}$ of $24 \mathrm{kG}$ and an $H_{k}$ of 20 Oe for the Fe-Co-N films, the FMR frequency determined from the Kittel equation is $1.94 \mathrm{GHz}$. 


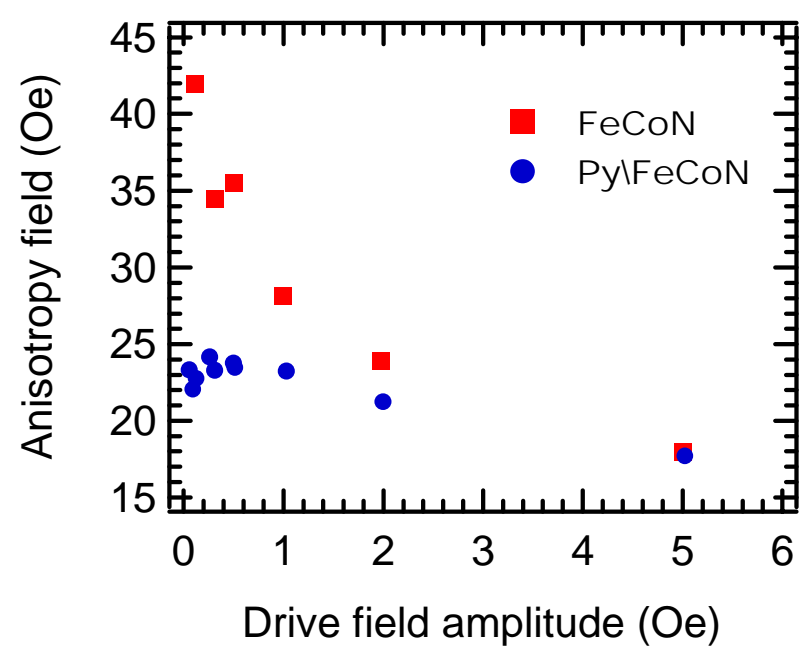

Figure 4. Anisotropy field for the Fe-Co-N film and the Py $\backslash \mathrm{Fe}-\mathrm{Co}-\mathrm{N}$ film as a function of the drive field amplitude

By putting a Permalloy nano-underlayer below the Fe-Co-N film, we are able to improve the magnetic softness of the Py $\backslash \mathrm{Fe}-\mathrm{Co}-\mathrm{N}$ films, reduce its dispersion angle, and decrease its damping constant, which results in an enhanced FMR frequency and a greater bandwidth that are desired for applications in magnetic write heads and integrated inductors.

A major challenge for this work is that the new magnetic material must be deposited on polyimide, which covers the coils of an integrated inductor. Similarly, for fabricating magnetic recording heads, magnetic pole pieces sometimes must be deposited on hard cured photoresist or polyimide. Our work so far indicates that the Py\Fe-Co-N\Py films, when deposited on polyimide, retain their high permeability at high frequencies, as shown in Fig. 5, which is very encouraging for future device work incorporating the Fe-Co-N-based materials.

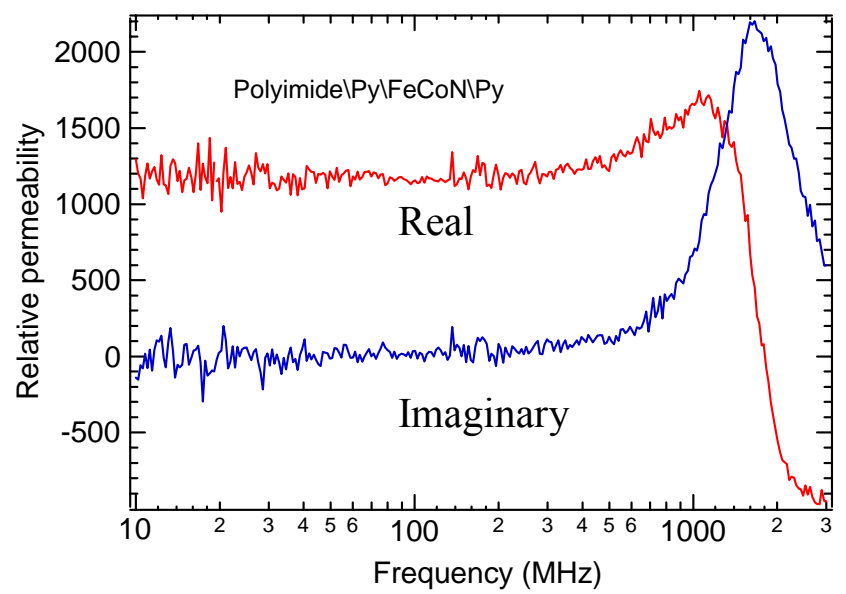

Figure 5. Real and imaginary permeability spectra of Py $\backslash$ Fe-Co-N $\backslash P y$ deposited on polyimide. 


\section{INTEGRATED INDUCTORS WITH Co-Ta-Zr MAGNETIC MATERIALS}

There have been significant efforts made to develop high resistivity and high anisotropy magnetic materials for RF applications [16-20]. Selection of a material for integrated inductors requires that the magnetic properties be stable up to a temperature of $400^{\circ} \mathrm{C}$, have high resistivity and a ferromagnetic resonance frequency beyond $1 \mathrm{GHz}$. One of the most suitable candidate was Co-4.5\%Ta-4.0\%Zr (at.\%) which exhibits a permeability of $\sim 870$, a resistivity of $\sim 100 \mu \Omega-\mathrm{cm}$ and a ferromagnetic resonance of $1.4 \mathrm{GHz}$. The alloy exhibits a relatively high saturation magnetization $4 \pi \mathrm{M}_{\mathrm{s}}$ of $15 \mathrm{kG}$, required because currents for power delivery applications are large and the inductance decreases rapidly as the magnetic material saturates. As-deposited CoTaZr also exhibits a relatively high anisotropy field, $\mathrm{H}_{\mathrm{k}}$, of $18 \mathrm{Oe}$, necessary to achieve a ferromagnetic resonance in the $\mathrm{GHz}$ frequency regime. The high anisotropy was achieved by depositing the material in a Veeco-CVC DC magnetron sputtering system using an applied switching magnetic field during the deposition.

The unique and most pertinent characteristic of CoTaZr is its thermal stability. Properties were measured before and after a $400^{\circ} \mathrm{C}$ PECVD oxide deposition, the highest temperature the material is exposed to during processing; magnetic properties remained the same after the annealing process.

An inductor is defined by the number of turns, $n$, the diameter of the inductor, $\mathrm{d}_{\text {out }}$, the wire width, w, and the spacing between wires, s. Planar octagonal and square spiral inductors were fabricated: a) with a solid magnetic ground plane, b) with a patterned (with slots) magnetic ground plane, and c) without a magnetic ground plane (Figure 6). The inductors are fabricated on $2 \mu \mathrm{m}$ of $\mathrm{SiO}_{2}$ grown on a silicon substrate. An underpass connects the incoming current to the center of the spiral and is made of magnetic material. The spiral conductor is made of $1 \mu \mathrm{m}$ of $\mathrm{Al}-\mathrm{Cu}$ and is isolated from the magnetic ground planes by $0.5 \mu \mathrm{m}$ or $1 \mu \mathrm{m}$ of $\mathrm{SiO}_{2}$. Since this oxide thickness determines the capacitance, it plays a key role in determining the resonance frequency of the inductor. The thickness of the magnetic ground planes was $0.2,0.4$ and $1 \mu \mathrm{m}$. For comparison purposes inductors with $1 \mu \mathrm{m}$ of $\mathrm{Al}-\mathrm{Cu}$ ground planes were also fabricated.
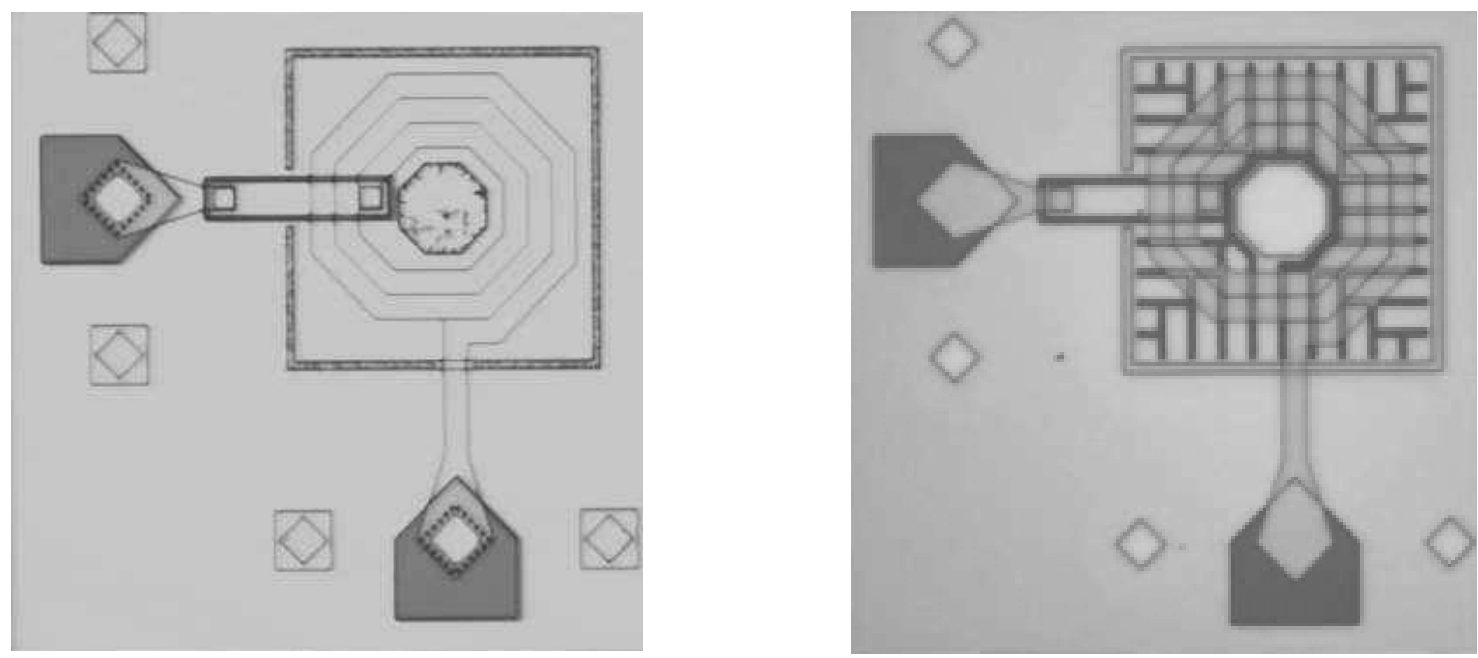

Figure 6. Pictures of 2.75-turn octagonal inductors: left, solid magnetic films; right, slotted magnetic films (to reduce eddy currents). 
As shown in Fig. 7, inductors fabricated using standard processing techniques have shown $60 \%$ increase in inductance over air core $(\sim 10 \mathrm{nH}$ versus $\sim 6 \mathrm{nH})$ due to the addition of a $1 \mu \mathrm{m}$ thick magnetic ground plane $(\mathrm{Co}-4.5 \% \mathrm{Ta}-4 \% \mathrm{Zr})$ below the inductor. The cutoff frequency is $>1 \mathrm{GHz}$. The magnetic materials are amorphous (as-deposited) using a CVC-Veeco sputtering system and have soft properties $\left(\mathrm{H}_{\mathrm{k}}=18 \mathrm{Oe}, \mathrm{H}_{\mathrm{c}}=0.2 \mathrm{Oe}, \mu_{\mathrm{r}}=870\right)$. CoTaZr shows no degradation of properties even after exposure to $400^{\circ} \mathrm{C}$. Among the devices we have fabricated so far, the highest frequency inductor has a $0.4 \mu \mathrm{m}-\mathrm{CoTaZr}$ ground plane with 2.75 turns in octagonal shape; it has a cutoff frequency of $3.3 \mathrm{GHz}$ (not shown here). In general the slotted devices have lower inductance yet higher frequency response due to lack of eddy current formation. It can be concluded that CoTaZr is a good candidate for high frequency integrated applications if the drive current is not so large as to saturate CoTaZr materials. Otherwise, high saturation materials such as $\mathrm{FeCoN}$ should be used.

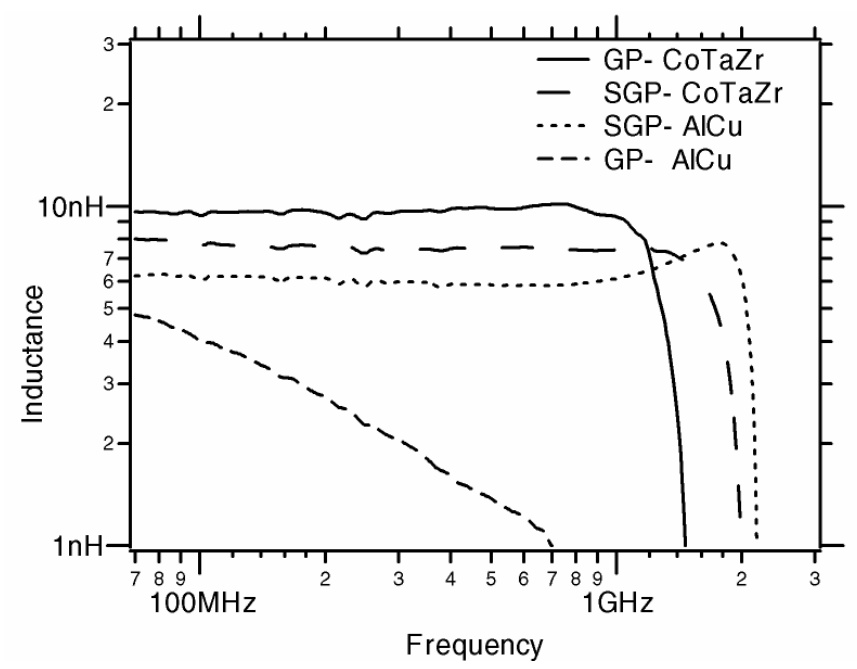

Figure 7. Inductance vs. Frequency for 3.75 turn octagonal inductors with solid ground planes (GP) and slotted ground planes (SGP).

The largest inductance enhancement is obtained when a conductor is enveloped within two layers of magnetic material. This behavior is attributed to flux closure around the conductor.

\section{ACKNOWLEDGMENTS}

The authors are indebted to Dr. T. J. Silva and A. B. Kos at the National Institute of Standards and Technology (NIST) on the PIMM measurements. Drs. Chin-Ya Hung, Yingjian Chen, Dean Rao, Syed Hossain, Chester X. Chien and Hua-Ching Tong for kindly providing the accessibility to the laboratory facilities at Read-Rite Corporation, and for their helpful discussions. A. M. C and S. X. Wang are indebted to D. Gardner, G. Vandentop, G. Marcyk, S. List, J. Chow, M. Ho, A. Cobarrubia and J. Talens at Intel, and J. Kools, M. Mao, F. Dahmani and M. Gutkin at CVC-Veeco for their generous assistance on the inductor work. This work is supported in part by NSF under grant ECS-0096704 and by Intel Corp.

\section{REFERENCES}


1. S. H. Charap, P. L. Lu, Y. He, IEEE Trans. Magn. 33, 978 (1997).

2. D. Weller, A. Moser, IEEE Trans. Magn. 35, 4423, (1999).

3. S. X. Wang, A. M. Taratorin, Magnetic Information Storage Technology (Academic Press, Boston, MA, 1999), p. 4 \& p. 482.

4. G. Prinz, Science, 282, 1660 (1998).

5. J. M. De Teresa, A. Barthelemy, A. Fert, J. P. Contour, F. Montaigne, P. Seneor, Science, 286, 507, (1999).

6. R. M. Bozorth, Ferromagnetism (Van Nostrand Company, 1951; reissued by IEEE Press, New York, NY, 1993), p. 195 \& p. 441.

7. E. J. Yun, W. Win, and R. M. Walser, IEEE Trans. Magn., 32, 4535, 1996.

8. T. Osaka et al., Nature, 392, 796, 1998.

9. W. P. Jayasekara, S. X. Wang, M. H. Kryder, J. Appl. Phys., 79, 5880, 1996.

10. S. X. Wang, N. X. Sun, M. Yamaguchi, and S. Yabukami, Nature, 407, 150 (2000)

11. N. X. Sun and S. X. Wang, IEEE Trans. Magn., 36, 2506 (2000).

12. W. R. Beam and K. Y. Ahn, J. Appl. Phys. 34, 1561 (1963).

13. T. J. Silva, C. S. Lee, T. M. Crawford, and C. T. Rogers, J. Appl. Phys. 86, 7849 (1999).

14. N. X. Sun, S. X. Wang, T. J. Silva, and A. B. Kos, IEEE Trans. Magn.,38, 146 (2002).

15. C. Kittel, Introduction to Solid State Physics, p. 505. ( $7^{\text {th }}$ edition, John Willey and Sons, 1996)

16. J. Y. Park, S. H. Han, and M. G. Allen, IEEE Transactions on Magnetics 35, 4291 (1999).

17. M. Yamaguchi, K. Suezawa, K.I. Arai, Y. Takahashi, S. Kikuchi, Y. Shimada, W.D. Li, S. Tanabe, and K. Ito, J. Appl. Phys. 85, 5148 (1999).

18. V. Korenivski and R.B. van Dover, J. Appl. Phys. 10, 5247 (1997).

19. C. P. Yue, and S. S. Wong, IEEE J. Solid State Circuits 33, 743 (1998).

20. S.S Mohan, M. Hershenson, P.P. Boyd, and T.H. Lee, IEEE J. Solid State Phys. 34, 1419 (1999). 